[3] Galiè $\mathrm{N}$ et al. Eur Heart J 37:67-119, 2016.

[4] Coghlan JG et al. Ann Rheum Dis 73:1340-1349, 2014.

Disclosure of Interest: None declared

DOI: 10.1136/annrheumdis-2017-eular.4608

\section{SAT0344 DISEASE RELATED MALNUTRITION IN SYSTEMIC SCLEROSIS AND ASSOCIATED FACTORS: A CROSS-SECTIONAL STUDY}

I. Türk ${ }^{1}$, N. Cuzdan Coskun ${ }^{2}$, V. Ciftci ${ }^{3}$, D. Arslan Taş ${ }^{1}$, M.C. Doğan ${ }^{4}$, I. Unal ${ }^{5}$ ${ }^{1}$ Department of Internal Medicine, Division of Rheumatology; ${ }^{2}$ Department of Physical Medicine and Rehabilitation, Division of Rheumatology; ${ }^{3}$ Faculty of Dentistry, Department of Pediatric Dentistry; ${ }^{4}$ Department of Pediatric Dentistry; ${ }^{5}$ Department of Biostatistics, Çukurova University, Adana, Turkey

Background: The risk of malnutrition increases in patients with systemic sclerosis (SSc) which has a negative prognostic effect. Additionally malnutrition is a significant cause of morbidity and mortality.

Objectives: The aim of this study was to evaluate the associations between malnutrition and clinical features of the disease, depression in SSc patients.

Methods: Concomitant SSc patients followed in our outpatient and inpatient clinics were enrolled in the study. Skin involvement was assessed with modified Rodnan skin score (mRSS), joint/tendon involvement with finger-tip to palm distance (FTP). Interstitial lung disease (ILD) and heart involvement were evaluated with clinical and radiological methods. Patients were questioned for dysphagia and gastroesofageal reflux as an indicator of esophageal involvement, early satiety and vomiting as gastric involvement, and diarrhea, constipation and bloating as bowel involvement. ${ }^{1}$ Interincisal distance measurement was used to assess the maximal mouth opening capacity. Malnutrition risk was assessed by the Malnutrition universal screening tool (MUST). The Beck Depression Inventory (BDI) was used for measuring the severity of depression. sWe examined associations between malnutrition risk and clinical features of the disease, depression in SSc patients. Results: Ninety eight SSc patients with 69 diffuse and 29 limited type of the disease were enrolled in the study. $84.7 \%$ of the patients were female and the mean age was $52.67 \pm 11.28$ years. According to MUST scores $61.2 \%$ of patients have low, $15.3 \%$ medium and $23.5 \%$ high risk for malnutrition. We found no difference between the malnutrition risk among genders $(p=0.065)$. mRSS and FTP were significantly different between malnutrition risk groups $(p=0.005,0.050$ respectively). Malnutrition risk was higher with patients with ILD than the patients without ILD $(p=0.044)$. Malnutrition risk was higher with patients with bowel involvement than the patients without bowel involvement. $(p=0.021)$.

Interincisal distance was significantly different between malnutrition risk groups $(p=0.003) .78 .7 \%$ of SSc patients have BDI scores $\geq 10,54.3 \%$ of SSc patients have $\mathrm{BDI}$ scores $\geq 17$. BDI scores were significantly different between malnutrition risk groups $(p<0.001)$. Factors affecting malnutrition risk were evaluated with logistic regression analysis. Interincisal distance and bowel involvement were found to be the most relevant factors for malnutrition risk. SSc patients with bowel involvement have 2.519-fold increased risk of malnutrition compared to patients without bowel involvement (95\% Cl 1.039-6.105). Every $1 \mathrm{~mm}$ decrement in interincisal distance was associated with 1.101 fold increase in malnutrititon risk of SSc patients $(95 \% \mathrm{Cl} 1.032-1.176)$.

Conclusions: Malnutrition is common in SSc patients. Malnutrition risk is associated with skin, tendon/joint involvement, DU count, ILD, bowel involvement, microstomia and depression severity.

\section{References:}

[1] Walker UA, Tyndall A, Czirják $L$ et al. Clinical risk assessment of organ manifestations in systemic sclerosis: a report from the EULAR Scleroderma Trials And Research group database. Ann Rheum Dis. 2007;66:754-63.

Disclosure of Interest: None declared

DOI: 10.1136/annrheumdis-2017-eular.6162

\section{SAT0345 THE IMPACT OF GASTROINTESTINAL INVOLVEMENT IN HEALTH-RELATED QUALITY OF LIFE IN PATIENTS WITH SYSTEMIC SCLEROSIS}

J. Caetano ${ }^{1}$, J. Branco ${ }^{2}$, S. Oliveira ${ }^{1}$, J. Delgado Alves ${ }^{1,3} \cdot{ }^{1}$ Systemic Autoimmune Diseases Unit, Department of Medicine IV; ${ }^{2}$ Department of Gastroenterology, Fernando Fonseca Hospital, Amadora; ${ }^{3}$ CEDOC, NOVA Medical School, Lisbon, Portugal

Background: Apart from skin involvement, the gastrointestinal (Gl) system is the second most commonly involved organ in systemic sclerosis (SSc), affecting over $80 \%$ of the patients. Although rarely being a direct cause of death, it is associated to a high morbidity and to a significant impairment in health-related quality of life (HRQoL). Few data are published considering the influence of GI manifestations in the quality of life of SSc patients.

Objectives: The purpose of this study is to characterize the impact of Gl involvement in HRQoL in SSc, and to compare it with that in patients with inflammatory bowel disease (IBD).

Methods: 29 consecutive SSc patients with GI involvement were selected from a cohort of 44 SSc patients, followed in a single referral centre and classified according to 2013 ACR/EULAR criteria. Comparative analysis was made with 24 consecutive patients with IBD without arthritis (14 Crohn's disease; 10 - ulcerative colitis). Health Assessment Questionnaire (HAQ-DI) and Short Form 36 (SF36), physical component summary (PCS) and mental component summary (MCS) scales, were used to assess HRQoL in both groups. The UCLA Scleroderma Clinical Trial Consortium Gastrointestinal Tract 2.0 (UCLA SCTG GIT 2.0) was performed in SSc patients to assess the severity of $\mathrm{Gl}$ involvement. Clinical data were obtained by medical records review. T-test and Fisher's exact test were used to compare binary variables. Pearson's correlation was used for continuous variables.

Results: The most common Gl segments involved in SSc patients were the esophagus in $90 \%$, the stomach in $60 \%$ and the bowel in $48 \%$, while the anorectum was involved in a smaller percentage (10.3\%). The mean UCLA SCTG GIT 2.0 score was $0.64 \pm 0.51$, compatible with a moderate severity, with higher scores obtained for reflux, distention and emotional wellbeing. The mean HAQ-DI score was 1.13 \pm 0.57 (0-best health), and the mean PCS and MCS scores were $35.2 \pm 9.4$ and $35.3 \pm 11.4$ (100-best health), respectively. These scores were significantly worse than in SSc patients without GI involvement (HAQ-DI $p=0.001$; PCS $-p=0.04$ and MCS $-p=0.005$ ). There was a significant correlation between higher UCLA SCTG GIT 2.0 scores and worse quality of life evaluated by HAQ-DI $(r=0.42, p=0.03)$, but the same correlation was not found for the SF36 components. Comparing with IBD patients, SSc patients with Gl involvement had worse life quality, with statistical significantly higher HAQ-DI $(p<0.001)$ and lower PCS $(p<0.001)$ and MCS $(p=0.01)$ scores.

Conclusions: Gl involvement in SSc significantly impaired patient's quality of life. The impact of Gl involvement in HRQoL of SSc patients was more severe than in IBD patients. Although physical components were relevant, with esophagus being most frequently involved, mental components associated with Gl involvement significantly compromised HRQoL in SSc. Therefore, the assessment of SSc patients using clinical severity measure tools, similar to UCLA SCTG GIT 2.0 score, is crucial for a better characterization of the disease and to an optimized clinical approach.

Disclosure of Interest: None declared

DOI: 10.1136/annrheumdis-2017-eular.2496

\section{SAT0346 SYSTEMIC SCLEROSIS'S EARLY STAGES AND ITS SIGNIFICANCE IN DAILY PRACTICE}

J.P. Abreu, I. Almeida, A. Marinho, C. Vasconcelos. Unidade de Imunologia Clínica, Centro Hospitalar do Porto, Porto, Portugal

Background: Systemic Sclerosis (SSc) is an autoimmune disease, characterized by skin and internal organs fibrosis. Its clinical manifestations are heterogeneous, hence, the definitive diagnosis of SSc rely on scores, such as American College of Rheumatology (ACR) for SSc. Leroy and Medsger, proposed the potential criteria for the classification of early stages of SSc, due to the insufficient sensitivity previous methods. Lately EUSTAR proposed the concept of Very Early SSc, defined by the presence of 3 red flag - RF, Puffy Fingers and ANA; if 2 of these are present, capilaroscopy and specific antibody must be accessed; if one of the last positive, further search for Systemic involvement must be taken in account. Objectives: We propose to: analyse a group of patients that had been diagnosed with early stages of SSc; study the presence of systemic involvement (at the time of diagnosis and throughout the follow-up), and the possible predictors for progression to definitive SSc.

Methods: The patients were selected by consulting the clinical data from patients followed in our hospital with diagnose of early stages of SSc. Very Early SSc criteria were used to define early stage SSc. The data concerning red flags, capilaroscopy, specific auto-antibodies, systemic involvement (Gastrointestinal, Pulmonary, Cardiac, Kidney, Muscoloskeletal).

Results: We obtained a sample of 70 patients, 65 being female subjects $(92,9 \%)$, with mean age of diagnosis 47.9 years (SD 13,6). 16 patients $(22,9 \%)$ were classified as Pre-scleroderma at the admission, 16 as Early SSc (22,9\%), 15 $(21,4 \%)$ as Very Early SSc, $16(22,9 \%)$ as possible SSc, $6(8,6 \%)$ as Limited SSc (despite not scoring to ACR criteria) and $1(1,4 \%)$ as Sin Scleroderma SSc. 63 patients $(90 \%)$ had RP, $15(21,4 \%)$ had puffy fingers, $63(90 \%)$ ANA positivity 11 patients $(15,7 \%)$ had 1 red flag, $45(64,3 \%)$ had 2 red flags and $13(18,6 \%)$ had 3 red flags.

54 patients $(78.3 \%)$ were positive to specific SSc autoantibodies; 47 patients $(71.2 \%)$ had capiloroscopy compatible with SSc in different stages $(17.1 \%$ Early, $12,9 \%$ Active, $4,3 \%$ Late and $32,9 \%$ compatible). 54 patients $(77,1 \%)$ scored for Very Early SSc.

At the moment of the diagnosis, 56 patient $(81,2 \%)$ had systemic involvement. 34 patients $(48,6 \%)$ scored to the definitive diagnosis by ACR 2013 criteria, after 2,56 years in mean (SD 2.51) of first diagnosis.

No correlation was found in what concerns to predictors of progression to SSc or systemic involvement.

Conclusions: This study shed a light on the importance of the recognition of SSc's early stages, since a significant part of these patients have systemic involvement at the moment of diagnosis, yet not scoring to ACR 2013 criteria. Almost half of the patients scored to definite diagnosis of SSc at the moment of the last appointment. The importance of these findings rely on the possibility to identify systemic involvement and address it in time to prevent/delay further disease progression.

References:

[1] Avouac J, Matucci-Cerinic M, Miniati I, Denton CP. Systemic Sclerosis. EULAR Online Course on Rheumatic Disease 2016 
[2] Varga J. Overview of the clinical manifestations of systemic sclerosis (scleroderma) in adults. UpToDate Jun 2016.

[3] LeRoy EC, Medsger TA Jr. Criteria for the classification of early systemic sclerosis. J Rheumatol 2001:28:1573-6.

[4] Amato L, Gallerani I, Berti S, Fabbri P. Prescleroderma: A Distinct Stage of Systemic Sclerosis. Skinmed. 2003; 2(1).

Disclosure of Interest: None declared

DOI: 10.1136/annrheumdis-2017-eular.6927

\section{SAT0347 DISEASE MODIFYING EFFECT OF ILOPROST IN PATIENTS WITH SYSTEMIC SCLEROSIS AND POSSIBLE ROLE OF CXCL4 CHEMOKINE}

K. Stefanantoni ${ }^{1}$, I. Sciarra ${ }^{1}$, M. Vasile ${ }^{1}$, R. Palazzo ${ }^{2}$, L. Frasca ${ }^{2}$, G. Valesini ${ }^{1}$, V. Riccieri ${ }^{1} .{ }^{1}$ Dipartimento di Medicina Interna e Specialità Mediche, Sapienza Università di Roma: ${ }^{2}$ National center for research and pre-clinical and clinical evaluation of drugs- Immune-mediated diseases UNIT, Istituto Superiore di Sanità (Nat. Inst. of Health), Rome, Italy

Background: lloprost is a synthetic prostaglandine used for vascular manifestation of Systemic Sclerosis (SSc), in particular it is indicated for active digital ulcers (DU) and severe Raynaud Phenomenon (RP) (1). It acts on several receptors such as IP and EP receptors and PPRs. Iloprost is also involved in the regulation of gene expression, fibrosis and inflammation $(2,3,4)$. CXCL4 o Platelet Factor 4 , is a 70KDa CXC-chemokine synthesized in megakaryocytes and plasmacytoid dendritic cells, released after platelet activation. In SSc, it seems to be higher in patients with early diffuse SSc and it correlates with mRSS and presence of PAH (5)

Objectives: To evaluate the effect of lloprost on vascular manifestations, on disease activity and on serum levels of CXCL4 at baseline (TO) and after 1 month (T1), 3 months (T3) and 6 months (T6) of therapy.

Methods: 30 patients $(\mathrm{M} / \mathrm{F}=1 / 29$; mean age $=58.2 \mathrm{yrs}$; mean disease duration $=12.8 \mathrm{yrs}$ ) with established SSc according to ACR criteria, were enrolled. At T0, $\mathrm{T} 1, \mathrm{~T} 3$ and T6 treatment with lloprost at standard dosage we determined RP VAS, number of DU, European Scleroderma Study Group Activity Index (EScSGAI) and serum levels of CXCL4, measured using commercially available immunoassay kit (Human CXCL4/PF4 R\&D SYSTEMS ${ }^{\circledR}$ ). All patients underwent to a complete clinical, instrumental and laboratory evaluation.

Results: Regarding RP VAS, we found a statistically significant increase $(p=0.04)$ at T3, corresponding to the colder winter period, while it decreased, although not significantly at $\mathrm{T} 1$ and at $\mathrm{T} 6$, where this reduction was significant compared to T3 values $(p=0.0008)$. Considering the number of active DU, we have highlighted the same trend of the RP VAS. EScSGAI values showed a statistically significant reduction ( $\mathrm{p}=0.03$ ) comparing T3 to T6. Regarding $C X C L 4$, we found significantly higher levels in SSc patients respect to a group of healthy controls $(H C)(p=0.047)$. No significant difference was found in serum levels of CXCL4 at TO, T1,T3 and T6. Evaluating patients with higher levels of CXCL4 at baseline, respect to the average of $\mathrm{HC}$ values (CXCL4>25,000 pg/ml) (11/30 patients), we found that 7 subjects had a significant improvement in disease activity at T6 evaluated by EScSGAI $(p=0.015)$. In these patients we also detected a significant reduction in T3 CXCL4 values $(p=0.043)$ persisting and at T6, although not reaching statistical significance. Moreover higher basal levels of CXCL4 in patients with disease duration less of 60 months $(p=0.05)$ and in patients with pericardial effusion $(p=0.037)$ were detected. Besides we found significantly lower levels of CXCL4 in patients with DU history $(p=0.049)$ and esophageal involvement $(p=0.008)$.

Conclusions: Our study confirms the efficacy of lloprost on vascular manifestation of SSc and also highlights its effect on the disease activity. Moreover this drug seems to influence, in a subgroup of patients, concentrations of CXCL4, an important chemokine involved in SSc pathogenesis that deserves further investigations.

References:

[1] Kowal-Bielecka O et al. Ann Rheum Dis. 2009 May;68(5):620-8.

[2] Clapp LH et al. Prostaglandins Other Lipid Mediat. 2015.

[3] Dorris SL et al. Mediators Inflamm. 2012;2012:926968.

[4] Zhu Y et al. Respir Res. 2010 Mar 20;11:34.

[5] Van Bon et al. N Engl J Med. 2014 Jan 30;370(5):433-43.

Disclosure of Interest: None declared

DOI: 10.1136/annrheumdis-2017-eular.5731

\section{SAT0348 ASSOCIATION OF VITAMIN D DEFICIENCY WITH REDUCED IL10-PRODUCING REGULATORY B CELLS IN SYSTEMIC SCLEROSIS}

A. Mavropoulos, C. Liaskos, A. Gkoutzourelas, C.G. Katsiari, D.P. Bogdanos, L.I. Sakkas. Rheumatology and Clinical Immunology, University of Thessaly, School of Health Sciences, LARISSA, Greece

Background: IL-10-producing regulatory B cells (Bregs), also known as B10 cells, are decreased and functionally impaired in patients with systemic sclerosis (SSc), particularly in those with SSc-associated interstitial lung disease (1). As serum 25-OH-vitamin D (vitD) levels are associated with clinical aspects in patients with SSc (2), we investigated whether there is any link between vitD levels and Breg levels.
Objectives: To assess whether or not vitD deficiency in SSc is associated with the percentages of circulating IL-10-producing Breg cells.

Methods: PBMCs and serum samples were isolated from 55 patients with systemic sclerosis. Serum VitD levels were measured using a commercially available sandwich ELISA kit. Phenotypic analysis of CD19, CD24, CD27, CD38 and intracellular expression of cytoplasmic IL-10 following bacterial CpG (ODN2006) and PMA/ionomycin stimulation was examined by flow cytometry using specific fluorochrome-conjugated monoclonal antibodies (BD Biosciences). Results: Systemic sclerosis patients were divided into two groups (vitD deficient or not) based on a serum concentration cut off value of $20 \mathrm{ng} / \mathrm{ml}$. The mean vitD levels in the deficient group were $14.11 \pm 3.6 \mathrm{ng} / \mathrm{ml}(\mathrm{n}=17)$ whereas the mean vitD levels in the non-deficient group were $37.5 \pm 12.9 \mathrm{ng} / \mathrm{ml}(\mathrm{n}=38)$. IL-10-producing $B$ cells (B10 cells) were significantly decreased in vitD deficient patients compared to those with medium/high levels $(p=0.02)$. CD19+CD27+ (memory) $B$ cells were also significantly reduced in patients with VitD deficiency $(\mathrm{p}=0.004)$. In addition the ratio of naïve/memory $\mathrm{B}$ cells was significantly higher in VitD deficient patients $(p<0.05)$. Within the memory B cell fraction, the CD19+CD27+CD24hi cells also known as phenotypic memory Bregs, were mostly decreased $(p=0.001)$. There was no significant association between CD19+CD38hiCD24hi (transitional) Bregs and VitD levels.

Conclusions: Our data suggest that vitamin D deficiency may account for reduced $\mathrm{B} 10$ cells in systemic sclerosis

References:

[1] Mavropoulos et al Arthritis Rheumatol. 2016:68:494-504.

[2] Arnson $Y$ et al Autoimmun Rev. 2011;10:490-4.

Disclosure of Interest: None declared

DOI: 10.1136/annrheumdis-2017-eular.5747

\section{SAT0349 EVALUATION OF STANDARD SWALLOWING FUNCTION IN ASSESSING ASPIRATION RISK FOR PATIENTS WITH SYSTEMIC SCLEROSIS}

L. Yan, P. Yunfeng, Y. Mengru. Department of Rheumatology, Third Affiliated Hospital of Sun Yat-sen University, Guangzhou, China

Background: Swallowing dysfunction is a common symptom of systemic sclerosis, which usually induce aspiration pneumonia to aggravate lung progress. It is necessary to screen swallowing disorders effectively at an early stage.

Objectives: To investigate the value of standard swallowing function assessment (SSA) in aspiration screening for inpatients with Systemic sclerosis.

Methods: SSA and Video fluoroscopic swallowing study (VFSS) were performed in 120 inpatients with Systemic sclerosis from March 2014 to September 2016. The sensitivity, specificity, positive predictive value and negative predictive value of SSA in the diagnosis of aspiration in patients were calculated by taking VFSS examination as the gold standard. The incidence of pneumonia in SSA-positive and SSA-negative patients were compared.

Results: The diagnostic sensitivity of SSA was $81.2 \%$, the specificity was $75 \%$, the positive predictive value was $68.6 \%$ and the negative predictive value was $90.3 \%$. The incidence of pneumonia in SSA positive patients was higher than that in SSA negative patients $(54.1 \%$ vs $28.3 \%)(\mathrm{P}<0.01)$.

Conclusions: SSA is a valuable screening tool for the evaluation of aspiration risk in patients with Systemic sclerosis.

References:

[1] Systemic Sclerosis with Multiple Pulmonary Manifestations. Kotnur MR, Suresh P, Reddy VS, Sharma T, Salim NA. J Clin Diagn Res. 2016 Jun;10(6):OD16-7. doi: 10.7860/JCDR/2016/18781.8032.

[2] Progression of esophageal dysmotility in systemic sclerosis. Vischio J, Saeed F, Karimeddini M, Mubashir A, Feinn R, Caldito G, Striegel K, Rothfield N. J Rheumatol. 2012 May;39(5):986-91. doi: 10.3899/jrheum.110923.

Disclosure of Interest: None declared

DOI: 10.1136/annrheumdis-2017-eular.5441

\section{SAT0350 DOES A SYSTEMIC SCLEROSIS PATIENT'S CLINICAL PHENOTYPE DEMONSTRATE HIS AUTOANTIBODY STATUS?}

M. Boonstra ${ }^{1}$, J.A. Bakker ${ }^{2}$, M.K. Ninaber ${ }^{3}$, N. Ajmone Marsan ${ }^{4}$, T.W. Huizinga ${ }^{1}$, J.K. de Vries-Bouwstra ${ }^{1}$. ${ }^{1}$ Rheumatology; ${ }^{2}$ Clinical Chemistry and Laboratory Medicine; ${ }^{3}$ Pulmonology; ${ }^{4}$ Cardiology, LUMC, Leiden, Netherlands

Background: Although antibody status has shown to improve clinical subsetting in Systemic sclerosis (SSc), it is far from perfect. For optimal clinical subsetting as well as for evaluating the possible pathogenic role of SSc specific antibodies, it is relevant to know to what extent clinical SSc phenotypes are determined by presence of these antibodies.

Objectives: To evaluate 1. if clinical relevant subsets of SSc patients are distinguishable using only clinical data, 2. how SSc specific autoantibodies are distributed among these subsets, and 3 . whether adding antibody status to cluster analyses improves recognition of SSc subsets.

Methods: Using data from SSc patients of the Combined Care In Systemic Sclerosis (CCIS) cohort, Leiden University Medical Center, hierarchical clustering based on Ward Method was performed on the first obtained factor of principal component analysis of 7 organ systems (skin;lung;heart;kidney;muscle;vascular;gastro- 